\title{
Investigations on Adaptive Beamforming for Linear and Planar Smart Antenna Arrays using Sample Matrix Inversion Algorithm
}

\author{
Kaustabh Ghatak, \\ School of Electronics \\ Engineering, KIIT University \\ Bhubaneswar 751024, Odisha, \\ India
}

\author{
Anupama Senapati \\ School of Electronics \\ Engineering, KIIT University \\ Bhubaneswar 751024, Odisha, \\ India
}

\author{
Jibendu Sekhar Roy \\ School of Electronics \\ Engineering, KIIT University \\ Bhubaneswar 751024, Odisha, \\ India
}

\begin{abstract}
In this paper sample matrix inversion (SMI) algorithm is used to generate beam for a smart antenna. The capability of adaptive beam generation by SMI algorithm is investigated for linear and planar arrays. The accuracies of main beam toward user and null toward interferer, beam width and side lobe level with respect to the variation of block length are reported.
\end{abstract}

\section{General Terms}

Smart antenna, SMI algorithm

\section{Keywords}

Smart antenna; side lobe level; SMI algorithm; linear antenna array; planar antenna array

\section{INTRODUCTION}

The performance in mobile communication can be improved by adaptive smart antenna which process the signal adaptively based on the direction of arrival (DOA). Smart antenna not only generates beams toward the desired user but also produces null toward the undesired interferer. A large number of publications are available [1-8] where various types of algorithms are used for beamforming of smart antenna. Beamforming algorithms include least mean square (LMS) and its variants, recursive least square (RLS) algorithm, sample matrix inversion (SMI) algorithm etc. Applications of these algorithms are described in literature for the accuracies of main beam direction and null direction [1-8]. The least mean square (LMS) adaptive beamforming algorithm requires a large number of iterations before satisfactory convergence is achieved. In case of rapidly changing signal characteristics, LMS algorithm may not allow tracking of the desired signal in a satisfactory way. The eigen value spread of the array correlation matrix determines the rate of convergence of the weights. Relatively slow convergence of the LMS scheme may be circumvented using SMI algorithm.

In this paper, SMI algorithm is used to generate beam at for linear and planar smart antenna arrays. Variation of beamwidth and side lobe level for different values of block lengths $(\mathrm{K})$, are investigated.

\section{SAMPLE MATRIX INVERSION (SMI) ALGORITHM}

Sample matrix inversion algorithm is discontinuous adaptive algorithms. Sample matrix is a Time average estimate of the array correlation matrix using k-time samples. For ergodic random process, the time average estimate will be equal the actual correlation matrix. It's used in discontinuous transmission, however it requires the number of interferers and their positions remain constant during the duration of the block acquisition. SMI has faster conversion rate since it employs direct inversion of the covariance matrix. The block diagram of SMI is shown in Figure 1.

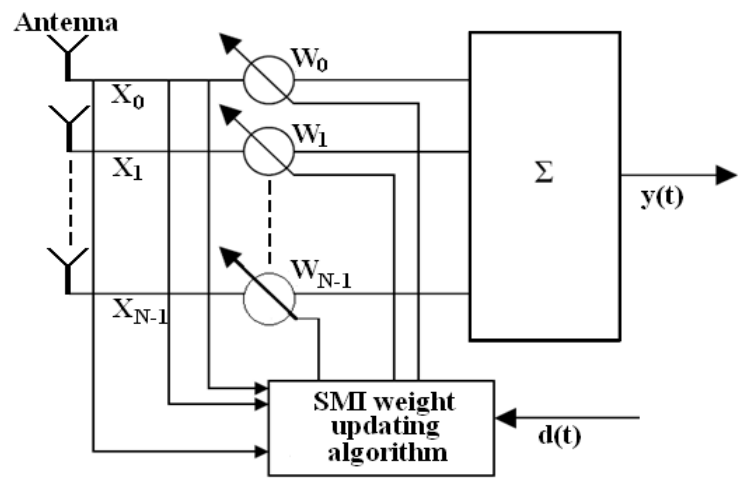

Fig 1: Sample matrix inversion adaptive beamforming network

One of the drawbacks of the LMS adaptive scheme is that the algorithm must go through many iterations before satisfactory convergence is achieved. SMI algorithm uses a block adaptive approach which would give a better performance than a continuous approach. The SMI has a faster convergence rate because it exploits the direct inversion of the covariance matrix. Sample matrix is a time average estimate of the array correlation matrix using K-time samples. The sample matrix is defined as the time average estimate of the array correlation, which uses $\mathrm{N}$ samples, and if the random process is ergodic in correlation, then time average estimate is equal to the real correlation matrix $[9,10]$ :

$\mathrm{R}_{\mathrm{xx}} \approx \frac{1}{N} \sum_{n=1}^{N} \mathrm{x}(\mathrm{n}) \mathrm{x}^{\mathrm{H}}(\mathrm{n})$

$\mathrm{r}=\frac{1}{N} \sum_{n=1}^{N} \mathrm{~d}^{*}(\mathrm{n}) \mathrm{x}(\mathrm{n})$

The matrix $x_{N}(n)$ is defined as the $\mathrm{n}$-th block of vectors $x$ ranges over $\mathrm{N}$-data snapshots:

$\mathrm{x}_{\mathrm{N}}(\mathrm{n})=\left[\begin{array}{cccc}\mathrm{x}_{1}(1+\mathrm{nN}) & \mathrm{x}_{1}(2+\mathrm{nN}) & \cdots & \mathrm{x}_{1}(\mathrm{~N}+\mathrm{nN}) \\ \vdots & \ddots & \vdots \\ \mathrm{x}_{\mathrm{N}}(1+\mathrm{nN}) & \mathrm{x}_{\mathrm{N}}(2+\mathrm{nN}) & \cdots & \mathrm{x}_{\mathrm{N}}(\mathrm{N}+\mathrm{nN})\end{array}\right]$

where, $\mathrm{n}$ represents the block number, and $\mathrm{N}$ is the block length. So, $R_{x x}$ can be given by

$\mathrm{R}_{\mathrm{xx}}(n)=\frac{1}{N} \mathrm{x}_{\mathrm{N}}(\mathrm{n}) \mathrm{x}_{\mathrm{N}}^{\mathrm{H}}(\mathrm{n})$

If the desired signal is 
$d(n)=[d(1+n K) d(2+n K) d(3+n K) \ldots \ldots \ldots d(N+$ $n K)$ ]

Then,

$\mathrm{r}=\frac{1}{\mathrm{~N}} \mathrm{~d}^{*}(\mathrm{n}) \mathrm{x}_{\mathrm{N}}(\mathrm{n})$

The sample matrix inversion weights of the $n$th block can be computed as

$w_{S M I}(n)=R_{x x}^{-1}(n) r(n)=\left[x_{N}(n) x_{N}^{H}(n)\right]^{-1} d^{*}(n) x_{N}(n)$

\section{BEAM GENERATION OF LINEAR SMART ANTENNA ARRAY USING SMI ALGORITHM}

Here, main beam and null at the desired directions of Linear Array are generated for the different values of block size (K) and comparing the results as beam direction, null direction, SLL minimum, Null-to-Null beamwidth (NNBW) for the SMI algorithm. Array factor for linear array is $[11,12]$

$\operatorname{AF}(\Phi)=\sum_{n=0}^{N-1} a_{n} \cdot \mathrm{e}^{((\operatorname{jn}(2 \pi \mathrm{d} / \lambda) \cos \Phi)+\alpha)}$

The progressive shift $\alpha$ is given by,

$\alpha=-\frac{2 \pi d}{\lambda} \cos \Phi_{0}, \Phi_{0}$ is the desired beam direction and $\lambda$ is the wavelength, element spacing $d=0.5 \lambda$, number of elements in the uniformed linear array, $\mathrm{N}=8$ and 40 , desired direction for main beam $=30^{\circ}$, desired direction for null $=60^{\circ}$.

Beams are generated for different values of block length $\mathrm{K}=50$ to $\mathrm{K}=1000$ and some of the results are for 8 -element linear array are plotted in Figure 2 and Figure 3. Normalized array factor means array factor divided by maximum value of array factor.

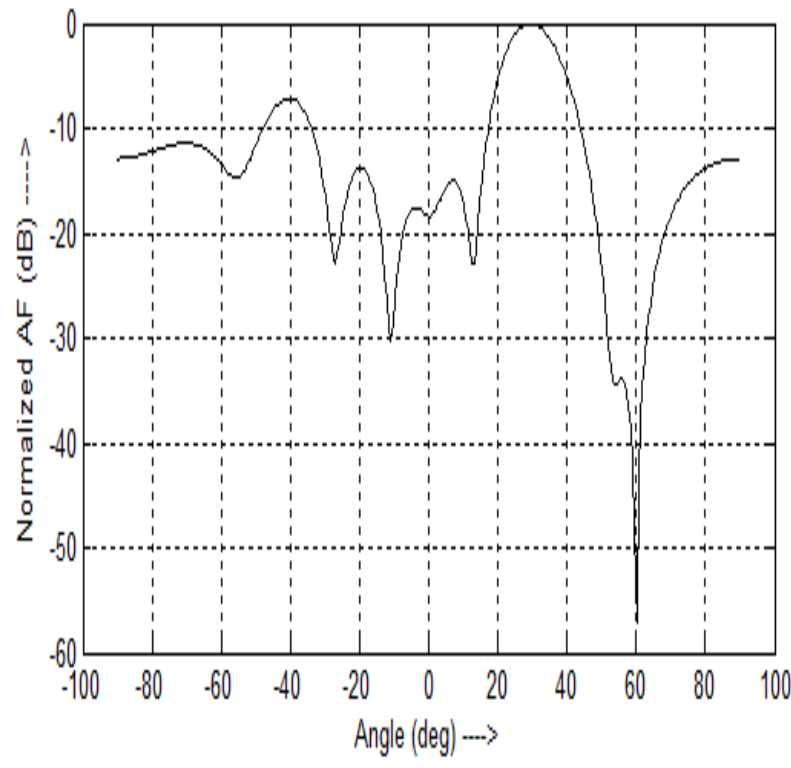

Fig 2: Radiation pattern for 8 elements linear array using SMI algorithm for $\mathrm{K}=50$

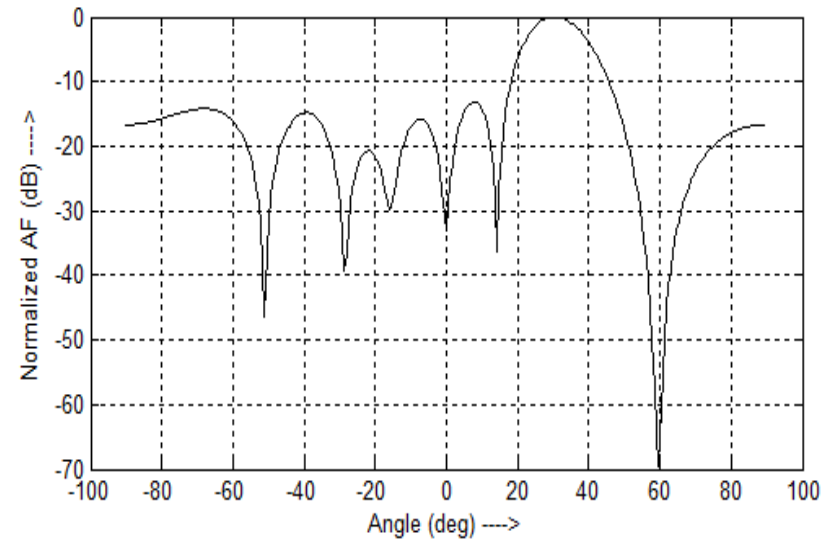

Fig 3: Radiation pattern for 8 elements linear array using SMI algorithm for $K=850$

Similarly, beams are generated for different values of block length $K=50$ to $K=1000$ and some of the results are for 40 element linear array are plotted in Figure 4 and Figure 5.

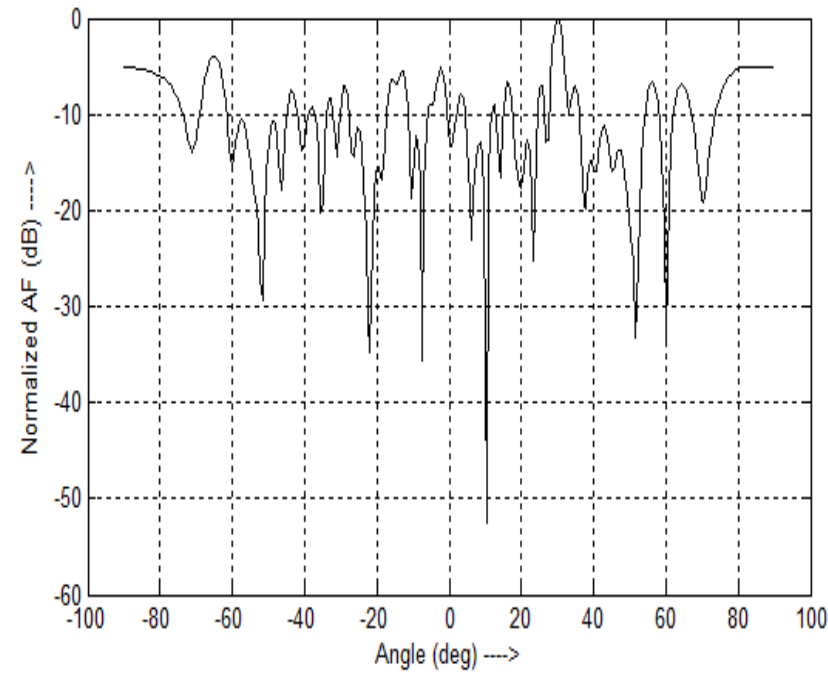

Fig 4: Radiation pattern for 40-elements linear array using SMI algorithm for $\mathrm{K}=50$

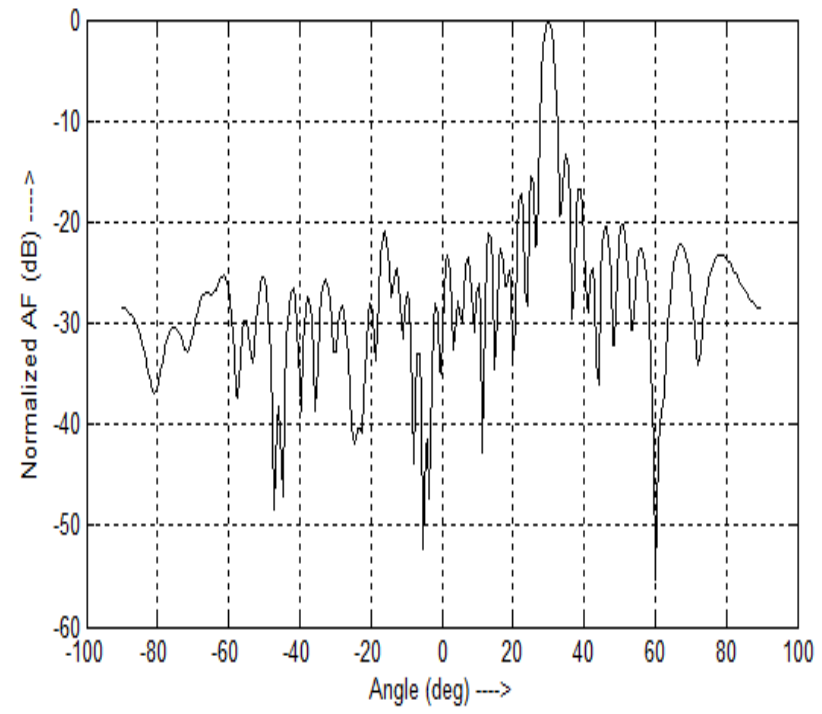

Fig 5: Radiation pattern for 40 -elements linear array using SMI algorithm for $K=850$ 
The results for different values of ' $\mathrm{K}$ ' are tabulated in Table 1 .

Table 1. Results for linear arrays

\begin{tabular}{|c|c|c|c|c|}
\hline \multirow{2}{*}{$\begin{array}{c}\text { Block } \\
\text { length } \\
(\mathrm{K})\end{array}$} & \multicolumn{2}{|c|}{ 8-element linear array } & \multicolumn{2}{|c|}{ 40-element linear array } \\
\hline & $\begin{array}{l}\text { Maximum } \\
\text { SLL (dB) }\end{array}$ & $\begin{array}{c}\text { Null-to- } \\
\text { null } \\
\text { beamwidth } \\
\text { (Deg) }\end{array}$ & $\begin{array}{l}\text { Maximum } \\
\text { SLL (dB) }\end{array}$ & $\begin{array}{c}\text { Null-to- } \\
\text { null } \\
\text { beamwidt } \\
\text { h (Deg) }\end{array}$ \\
\hline 50 & -7.16 & 36.67 & -3.967 & 5.73 \\
\hline 150 & -14.20 & 42.97 & -7.855 & 6.88 \\
\hline 200 & -14.69 & 42.39 & -9.982 & 7.30 \\
\hline 250 & -12.16 & 42.4 & -13.99 & 6.88 \\
\hline 350 & -13.12 & 41.25 & -11.19 & 6.31 \\
\hline 400 & -10.77 & 40.68 & -12.72 & 7.45 \\
\hline 450 & -13.11 & 39.53 & -14.68 & 6.88 \\
\hline 550 & -13.89 & 41.25 & -11.81 & 6.31 \\
\hline 600 & -11.43 & 40.68 & -11.33 & 6.88 \\
\hline 700 & -13.37 & 42.39 & -11.81 & 6.31 \\
\hline 800 & -12.52 & 41.25 & -11.97 & 6.3 \\
\hline 850 & -13.20 & 41.82 & -13.29 & 6.88 \\
\hline 900 & -13.81 & 43.54 & -11.99 & 5.73 \\
\hline 950 & -12.20 & 40.68 & -12.25 & 6.31 \\
\hline 1000 & -12.33 & 40.68 & -12.21 & 6.31 \\
\hline
\end{tabular}

\section{BEAM GENERATION OF PLANAR} SMART ANTENNA ARRAY USING SMI

\section{ALGORITHM}

Array factor for uniformly spaced planar antenna array may be expressed as $[11,12]$

$$
\begin{aligned}
A F & =A F_{x M} A F_{y N} \\
A^{x} & =\sum_{m=1}^{M} I_{m 1} e^{j(m-1)\left(k d_{x} \sin \theta \cos \Phi \phi+\beta_{\mathrm{x}}\right)} \\
\mathrm{AF}_{\mathrm{yN}} & =\sum_{n=1}^{N} \mathrm{I}_{\mathrm{n} 1} \mathrm{e}^{\mathrm{j}(\mathrm{n}-1)\left(\mathrm{kd}_{\mathrm{y}} \sin \theta \sin \Phi \phi+\beta_{\mathrm{y}}\right)}
\end{aligned}
$$

For a uniform planar array, $I_{n 1}=I_{n 2}=I_{0}$, for all $m$ and n, i.e., all elements have same excitation amplitudes. Element spacing in terms of wavelengths, $d_{x}=d_{y}=d=0.5 \lambda$.

Here, 8 X8 and 16X16 planar antenna arrays are considered. Desired direction for main beam $=30^{\circ}$, desired direction for null $=60^{\circ}$. Beams are generated for different values of block length $K=50$ to $K=1000$ and some of the results are $8 X 8$ and 16X16 planar antenna arrays are plotted in Figure 6- Figure 9.

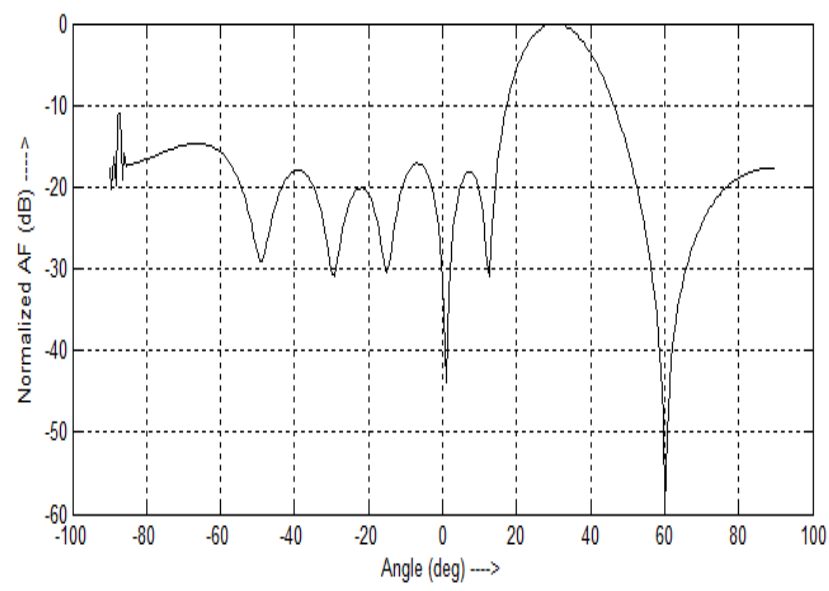

Fig 6: Radiation pattern for $8 X 8$ planar array using SMI algorithm for $K=150$

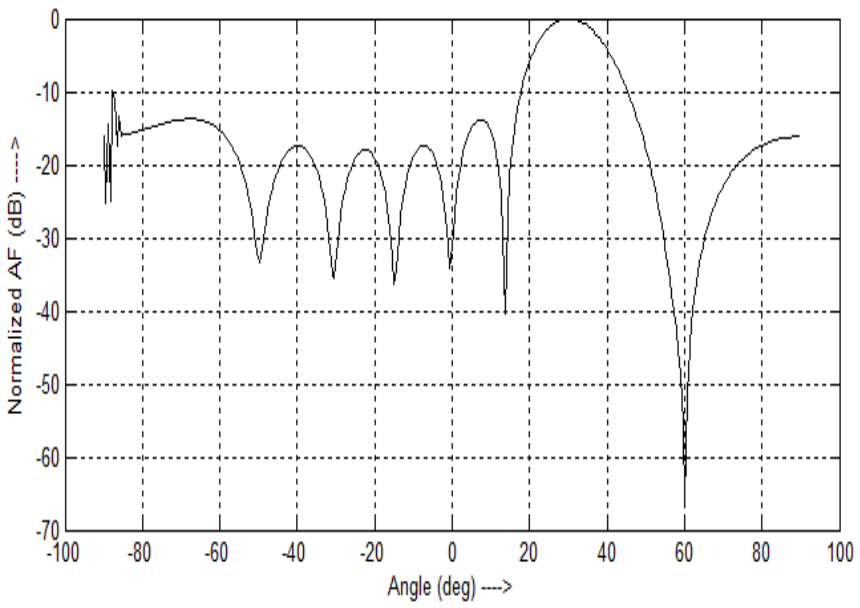

Fig 7: Radiation pattern for 8X8 planar array using SMI algorithm for $K=900$

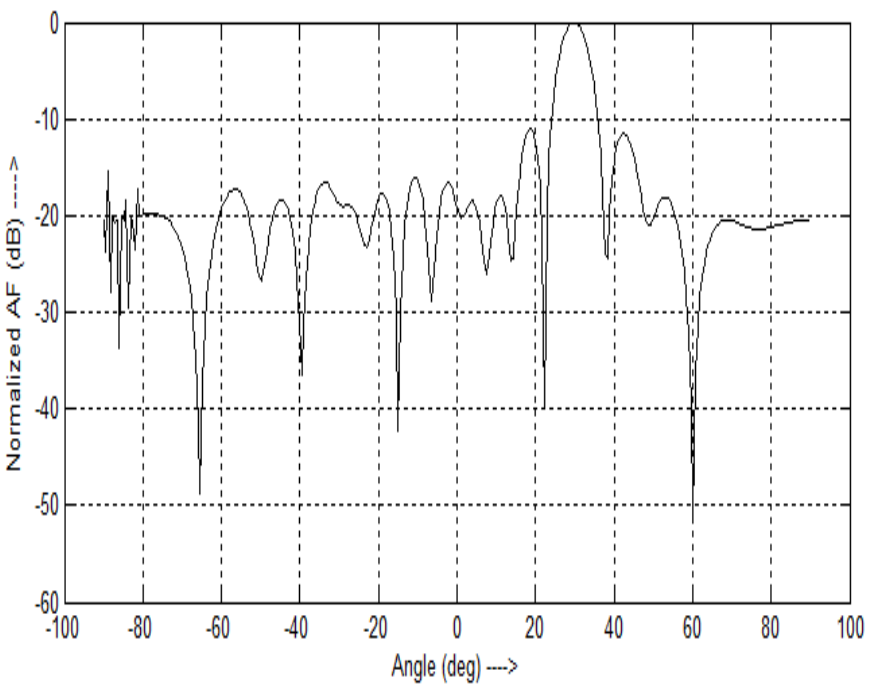

Fig 8: Radiation pattern for $16 X 16$ planar array using SMI algorithm for $K=150$ 


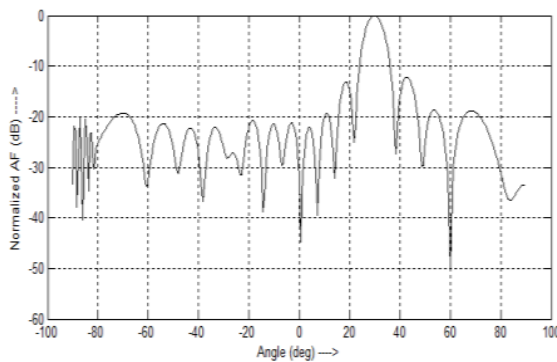

Fig 9: Radiation pattern for 16X16 planar array using SMI algorithm for $K=900$

The results for planar arrays are tabulated in Table 2. For planar array angle in $\theta$-direction and $\Phi$-direction are taken to be equal.

Table 2. Results for planar arrays

\begin{tabular}{|c|c|c|c|c|}
\hline \multirow{2}{*}{$\begin{array}{c}\text { Block } \\
\text { length } \\
(\mathrm{K})\end{array}$} & \multicolumn{2}{|c|}{$8 X 8$ planar array } & \multicolumn{2}{|c|}{ 16X16 planar array } \\
\hline & $\begin{array}{l}\text { Maximum } \\
\text { SLL (dB) }\end{array}$ & $\begin{array}{c}\text { Null-to- } \\
\text { null } \\
\text { beamwidt } \\
\text { h (Deg) }\end{array}$ & $\begin{array}{c}\text { Maximum } \\
\text { SLL(dB) }\end{array}$ & $\begin{array}{c}\text { Null-to-null } \\
\text { beamwidth } \\
\text { (Deg) }\end{array}$ \\
\hline 50 & -7.16 & 36.49 & -10.75 & 15.47 \\
\hline 150 & -17.21 & 44.12 & -11.08 & 16.61 \\
\hline 200 & -14.69 & 42.97 & -11.72 & 16.62 \\
\hline 250 & -12.21 & 42.97 & -10.95 & 16.61 \\
\hline 350 & -13.12 & 41.25 & -13.39 & 17.76 \\
\hline 400 & -10.77 & 40.68 & -12.16 & 16.04 \\
\hline 450 & -13.26 & 38.96 & -10.37 & 15.47 \\
\hline 550 & -13.89 & 40.68 & -11.49 & 15.47 \\
\hline 600 & -11.48 & 40.68 & -12.76 & 15.47 \\
\hline 650 & -13.67 & 40.10 & -12.52 & 17.73 \\
\hline 700 & -13.37 & 42.39 & -14.40 & 17.77 \\
\hline 800 & -12.46 & 41.25 & -12.66 & 16.04 \\
\hline 900 & -13.81 & 43.54 & -12.20 & 17.19 \\
\hline 950 & -12.20 & 40.68 & -11.78 & 16.04 \\
\hline 1000 & -12.23 & 40.68 & -13.27 & 16.04 \\
\hline
\end{tabular}

\section{DISCUSSION AND CONCLUSION}

SMI algorithm is applied for beamforming linear and planar smart antenna arrays. Block length ' $\mathrm{K}$ ' is varied from 50 to 1000. Accuracy of main bean direction and null direction do not depend on the value of ' $\mathrm{K}$ ', but beamwidth of main beam and side lobe level (SLL) depend on it. For 8-element linear array NNBW varies from $36.67^{\circ}$ to $42.97^{\circ}$, whereas for 40 element linear array this variation is very less, from $5.73^{0}$ to $7.45^{\circ}$. In this range of ' $\mathrm{K}$ ', for 8-element linear array variation of SLL is nearly $7 \mathrm{~dB}$ and for 40-element linear array this variation is about $10 \mathrm{~dB}$. For $8 \mathrm{X} 8$ planar array, variation of NNBW in this range of ' $\mathrm{K}$ ' is about $7^{0}$, whereas for $16 \mathrm{X} 16$ planar antenna array, it is about $1^{0}$. For $8 \mathrm{X} 8$ planar array, variation of SLL is about $10 \mathrm{~dB}$ and for $16 \mathrm{X} 16$ planar antenna array, it is about $4 \mathrm{~dB}$. Since reduced side lobe level is always preferred in communication, the appropriate value of block length is important for beam formation in smart antenna. The work may be extended using other beamforming algorithms, like, LMS and its variants, recursive least square (RLS) etc. Also combining those algorithms, like, LMS-SMI, NLMSSMI, SLMS-SMI a comparative study may be useful for research purpose.

\section{REFERENCES}

[1] Godara, L. C. 1997 Application of antenna arrays to mobile communications, Part II: beam-forming and direction-of-arrival considerations, Proceedings of the IEEE, vol. 85, no. 8, 1195-1245.

[2] Bellofiore, S., Balanis, C. A., Foutz , J. and Spanias, A. S. 2002 Smart-antenna systems for mobile communication network, part 1, overview and antenna design, IEEE Antennas and Propagation Magazine, vol. 44 , no. $3,145-154$.

[3] Sarkar, T. K., Wicks, M. C., Salazar-Palma M. and Bonneau, R. J. 2003 Smart Antennas, Wiley-IEEE Press.

[4] Mouhamadou, M., Vaudon, P. and Rammal, M. 2006 Smart antenna array patterns synthesis: null steering and multi-user beamforming by phase control, Progress In Electromagnetics Research (PIER), vol. 60, 95-106.

[5] Shubair, R.M. and Jassmi, W. 2006 Performance analysis of optimum SMI beamformers for spatial interference rejection, IEEE Personal Communications, 4743-4746.

[6] Ghouse Basha, T. S., Sridevi P. V. and Giri Prasad, M. N. 2013 Beam forming in smart antenna with precise direction of arrival estimation using MUSIC algorithm", Wireless Personal Communications, vol. 71, issue 2, 1353-1364.

[7] Tao J-W. and Chang, W-X. 2014 Adaptive beamforming based on complex quaternion processes, Mathematical Problems in Engineering, Hindawi, vol. 2014, 1-10.

[8] Senapati, A., Ghatak, K. and Roy, J. S. 2015 A Comparative study of adaptive beamforming techniques in smart antenna using LMS algorithm and its variants, International Conference on Computational Intelligence and Networks (CINE-2015), IEEE Xplore, KIIT University, Bhubaneswar, January 12-13, 58-62.

[9] Ali, W.A.E., Mohamed, D.A.E. and Hassan, A.H.G. 2013 Performance analysis of least mean square sample matrix inversion algorithm for smart antenna system, Loughborough Antennas \& Propagation Conference , IEEE Xplore, 624-629.

[10] Ali, W.A.E. and Hassan, A.H.G. 2014 A hybrid least mean square/sample matrix inversion algorithm using microstrip antenna array, Science and Information Conference, 871-876.

[11] Elliot, R. S. 2003 Antenna Theory and Design, Revised Edition, John Wiley, New Jersey.

[12] Balanis, C. A. 2005 Antenna Theory - Analysis and Design, $3^{\text {rd }}$ Ed., Wiley. 